\section{Short block codes for guaranteed convergence in soft-bit assisted iterative joint source and channel decoding}

Nasruminallah and L. Hanzo

Short block codes designed for guaranteed convergence in soft-bit assisted iterative joint source and channel decoding are proposed, which assist iterative soft-bit source decoding to attain an infinitesimally low bit error ratio.

Introduction: Soft-bit source decoding (SBSD) was proposed by Fingscheidt and Vary [1] for improving the convergence of iterative source-channel decoding (ISCD) [2] by exploiting the natural residual redundancy of the source-coded bit-stream. However, when using sophisticated state-of-the-art coding techniques, only modest residual redundancy is left in the source coded bit-stream. Therefore we propose to intentionally impose additional redundancy on the source coded bit-stream with the aid of the novel class of short block codes (SBCs) proposed.

The algorithm: The conventional SBSD scheme determines the extrinsic information from the natural residual redundancy, which remains in the bit-stream after source encoding. More explicitly, the sourceencoded bit-stream is partitioned into $M=2^{N}$-ary, or $N$-bit symbols, each of which has a different probability of occurance and will be termed as the information word encoded by the proposed SBC. We then characterise the redundancy of the source bit-stream with the aid of the non-uniform $M=2^{N}$-ary symbol probability distribution $P\left[s_{k}(\tau)\right]$, where $s_{k}(\tau)=\left[s^{k}(1), s^{k}(2), \ldots, s^{k}(M)\right]$, with $k=1,2, \ldots$, $N$ denoting the number of bits in each $M=2^{N}$-ary symbol. The details of the algorithm used for generating the extrinsic information using SBSD for the zero-order Markov model can be found in [2]. Provided that the bits of an $M=2^{N}$-ary symbol may be considered independent of each other, the channels' output information generated for the $\pi$ th $N$-bit symbol is given by the product of each of the constituent single-bit probabilities as follows:

$$
P\left[\hat{y}_{(k, \tau)} \mid y_{(k, \tau)}\right]=\prod_{i=1}^{N} P\left[\hat{y}(i)_{(k, \tau)} \mid y(i)_{(k, \tau)}\right]
$$

where $\hat{y}_{(k, \tau)}=\left[\hat{y}(1)_{k, \tau}, \hat{\tau}(2)_{k, \tau}, \ldots, \hat{y}(N)_{(k, \tau)}\right]$, is the received $N$-bit sequence. For each desired bit $\left[y(\lambda)_{(k, \tau)}\right]$, the extrinsic channel output information $P \hat{y}_{(k, \tau)}^{\text {[ext }} \mid y_{(k, \tau)}^{\text {[ext }]}$ is expressed as:

$$
P\left[\hat{y}_{(k, \tau)}^{[e x t]} \mid y_{(k, \tau)}^{[e x t]}\right]=\prod_{i=1, i \neq \lambda}^{N} P\left[\hat{y}(i)_{(k, \tau)} \mid y(i)_{(k, \tau)}\right]
$$

Finally, the resultant extrinsic logarithmic likelihood ratio (LLR) value can be obtained for each bit by combining its channel output information and the a priori knowledge of the corresponding $\tau$ th symbol as $[1,2]$ :

$$
\begin{aligned}
& \operatorname{LLR}\left[y(\lambda)_{k, \tau}\right]= \\
& \log \left(\frac{\sum_{k, \tau}^{[e x t]} P\left[y_{(k, \tau)}^{[e x t]} \mid y(\lambda)_{k, \tau}=+1\right] \cdot P\left[\hat{y}_{(k, \tau)} \mid y_{(k, \tau)}\right]}{\sum_{k, \tau}^{[e x t]} P\left[y_{(k, \tau)}^{[e x t]} \mid y(\lambda)_{k, \tau}=-1\right] \cdot P\left[\hat{y}_{(k, \tau)} \mid y_{(k, \tau)}\right]}\right)
\end{aligned}
$$

Using efficient state-of-the-art encoders, only limited source redundancy is left in the source coded bit-stream, which typically results in modest system performance improvements beyond two decoding iterations. Hence carefully controlled redundancy is imposed by the proposed rate $r=[N / N+1] \mathrm{SBCs}$ to ensure that the resultant $[N+1]$-bit codewords exhibit a minimum Hamming distance of $d_{H}=2$ between the $M=2^{N}$ number of legitimate $N$-bit source codewords, which was shown to be the necessary and sufficient condition for achieving the highest possible source entropy denoted as $H(X)=L_{S B S D}^{\text {extr }}=1$ bit, provided that the input a priori information of the SBSD is perfect, i.e. we have $H(X)=L_{S B S D}^{a p r i}=1$ bit [3]. According to our proposed SBC encoding procedure, first a redundant bit $r_{\tau}$ is generated for the $\pi$ th $M$-ary source word by calculating the XOR function of its $N$ constituent bits, according to $r_{\tau}=\left[b^{\tau}(1) \oplus b^{\tau}(2) \cdots \oplus b^{\tau}(N)\right]$; where $\oplus$ represents the XOR operation. The resultant redundant bit can be incorporated in any of the $[N+1]$ different bit positions of the resultant $\mathrm{SBC}$, in order to create $[N+1]$ different $\mathrm{SBC}$-encoded word combinations, as depicted in Table 1 , each having a minimum Hamming distance of $d_{H}=2$ from all the others.

Table 1: $[N+1]$ Different SBC combinations

\begin{tabular}{|l|c|c|c|c|}
\hline Symbols & $C_{1}$ & $C_{2}$ & $\cdots$ & $C_{N+1}$ \\
\hline$S_{(1)}$ & $r_{1} b_{1} b_{2} . . b_{N}$ & $b_{1} r_{1} b_{2} . . b_{N}$ & $\cdot$ & $b_{1} b_{2} . . b_{N} r_{1}$ \\
\hline$S_{(2)}$ & $r_{2} b_{1} b_{2} . . b_{N}$ & $b_{1} r_{2} b_{2} . . b_{N}$ & $\cdot$ & $b_{1} b_{2} . . b_{N} r_{2}$ \\
\hline$:$ & $:$ & $:$ & $:$ & $:$ \\
\hline$S_{(2 N)}$ & $r_{2^{N}} b_{1} b_{2} . . b_{N}$ & $b_{1} r_{2^{N}} b_{2} . . b_{N}$ & $\cdot$ & $b_{1} b_{2 . .} b_{N} r_{2^{N}}$ \\
\hline
\end{tabular}

Design example: The schematic of our proposed videophone arrangement used as our design example for quantifying the performance of various SBC schemes is shown in Fig. 1. At the transmitter side the video sequence is compressed using the H.264 video codec and the video source bit-stream $x_{k}$ is mapped or encoded into the bitstring $x_{m}^{\prime}$ using the specific bit-coding scheme of the SBC employed. Subsequently the output bit-string of the SBC is interleaved using the bit-interleaver $\Pi$ of Fig. 1, yielding the interleaved sequence $\bar{x}_{m}$, which is then encoded by a recursive systematic convolution (RSC) code having a specific code rate. The resultant bit-stream is QPSK modulated and transmitted over a correlated narrowband Rayleigh fading channel, associated with the normalised Doppler frequency of $f_{D}=0.01$. The received signal is QPSK demodulated and the resultant soft-information is passed to the RSC decoder. The extrinsic information gleaned is then exchanged between the SBSD and RSC decoders of Fig. 1, in order to attain the lowest possible bit error ratio (BER).

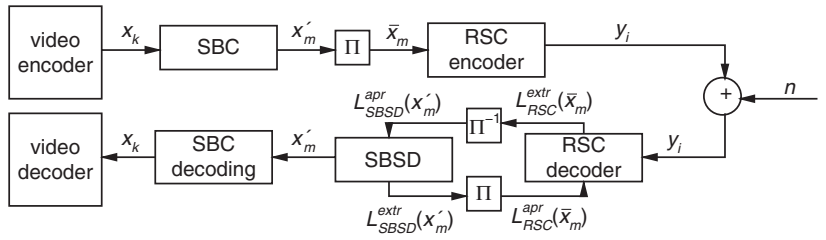

Fig. 1 Proposed system model for design example

Table 2: Code rates for different error protection schemes

\begin{tabular}{|l|c|c|c|c|c|c|c|}
\hline \multirow{2}{*}{ SBC } & \multicolumn{3}{|c|}{ Code rate } & \multirow{2}{*}{ SBC } & \multicolumn{3}{|c|}{ Code rate } \\
\cline { 2 - 5 } \cline { 7 - 8 } & RSC & SBC & Overall & & RSC & SBC & Overall \\
\hline Rate-1 & $1 / 2$ & 1 & $1 / 2$ & Rate-4/5 & $5 / 8$ & $4 / 5$ & $1 / 2$ \\
\hline Rate-2/3 & $3 / 4$ & $2 / 3$ & $1 / 2$ & Rate-5/6 & $3 / 5$ & $5 / 6$ & $1 / 2$ \\
\hline Rate-3/4 & $2 / 3$ & $3 / 4$ & $1 / 2$ & Rate-6/7 & $7 / 12$ & $6 / 7$ & $1 / 2$ \\
\hline
\end{tabular}

Results: The achievable system performance was evaluated using the 'Akiyo' video sequence [4] consisting of $45(176 \times 144)$ pixel quarter common intermediate format (QCIF) frames and encoded using the H.264/AVC JM 13.2 reference video codec at 15 framesper-second (fps) at the target bitrate of $64 \mathrm{kbit} / \mathrm{s}$. Each QCIF frame was partitioned into nine slices and each slice was composed of 11 macro-blocks (MBs) of a row of MBs within a QCIF frame. An intra-coded 'I' frame was inserted in the video sequence after every 45 frames, in order to curtail error propagation. Additionally, to control the effects of error propagation, we used intra-frame coded MB updates of three randomly dispersed MBs per frame. The coding parameters of the different SBC schemes is shown in Table 2. The overall code-rate of $R=1 / 2$ was maintained by adjusting the puncturing rate of the RSC in order to accommodate the different SBC rates of Table 2. The attainable BER performance using 2/3, 3/4, 4/5, 5/6, and $6 / 7$ rate $\mathrm{SBCs}$ is shown in Fig. 2. It is observed from Fig. 3 that SBSD operating in conjunction with rate-1 SBC results in an inferior PSNR performance in comparison to all the other SBC schemes having a rate lower than one, with an additional $\mathrm{E}_{b} / N_{0}$ gain of $20 \mathrm{~dB}$, relative to the rate-1 SBC, which in effect dispensed with the SBC. 


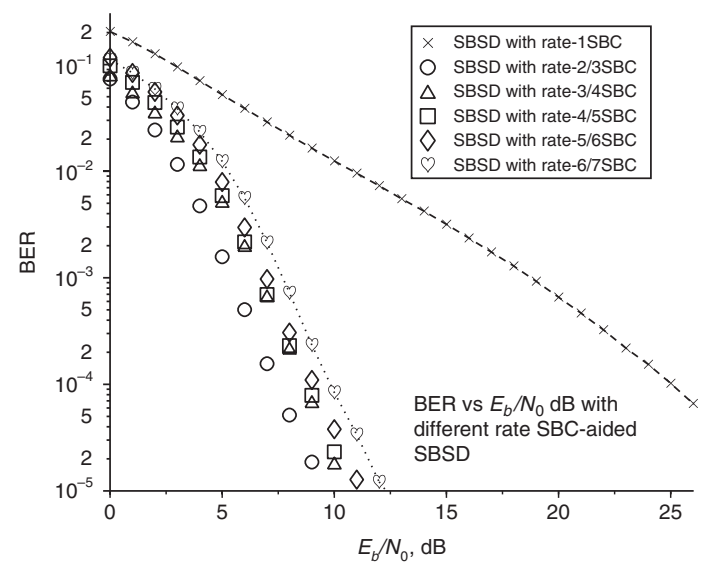

Fig. 2 BER performance of various error protection schemes

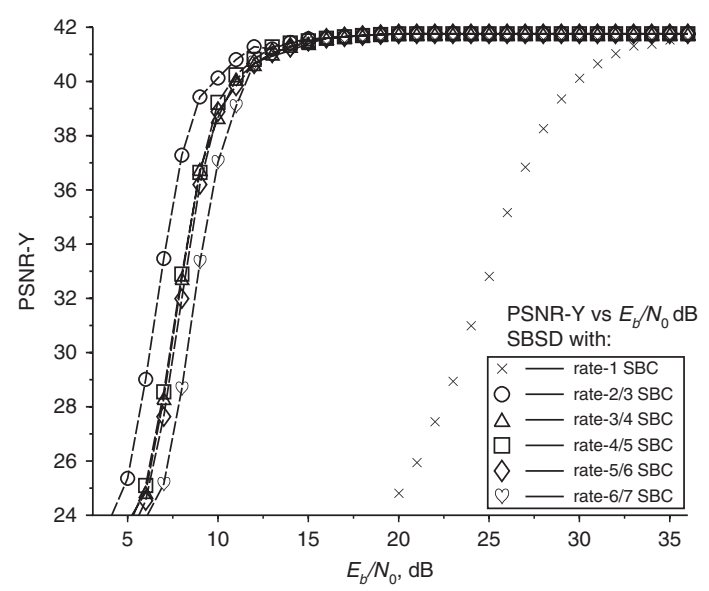

Fig. 3 PSNR-Y performance of various error protection schemes

Conclusion: Generic low-complexity SBCs are proposed for meeting the sufficient condition of $d_{H, \min }=2$ and hence guaranteeing decoding convergence for arbitrary SBSD-aided multimedia source codecs. The H.264/SBC/RSC design example exhibited a $20 \mathrm{~dB} E_{b} / N_{0}$ gain.
(C) The Institution of Engineering and Technology 2008

17 June 2008

Electronics Letters online no: 20081698

doi: 10.1049/el:20081698

Nasruminallah and L. Hanzo (School of ECS, University of Southampton, SO17 1BJ, United Kingdom)

E-mail: 1h@ecs.soton.ac.uk

\section{References}

1 Fingscheidt, T., and Vary, P.: 'Softbit speech decoding: a new approach to error concealment', IEEE Trans. Speech Audio Process., 2001, 9, pp. $240-251$

2 Adrat, M., and Vary, P.: 'Iterative source-channel decoding: improved system design using EXIT charts', EURASIP J. Appl. Signal Process., 2005, 2005, (1), pp. 928-941

3 Kliewer, J., Goertz, N., and Mertins, A.: 'Iterative source-channel decoding with Markov random field source models', IEEE Trans. Signal Process., 2006, 54, (10), pp. 3688-3701

4 L. Hanzo, P. Cherriman, and J. Streit: 'Video Compression and Communications: From Basics to H.261, H.263, H.264, MPEG2, MPEG4 for DVB and HSDPA-Style Adaptive Turbo-Transceivers' (Wiley-IEEE Press) http://eu.wiley.com/WileyCDA/WileyTitle/ productCd-0470518499.html, 2007 